\title{
A Hybrid Relay and Intelligent Reflecting Surface Network and its Ergodic Performance Analysis
}

\author{
Zaid Abdullah, Member, IEEE, Gaojie Chen, Senior Member, IEEE, Sangarapillai Lambotharan, Senior \\ Member, IEEE, and Jonathon A. Chambers, Fellow, IEEE
}

\begin{abstract}
This letter proposes a novel hybrid relay and Intelligent Reflecting Surface (IRS) assisted system for future wireless networks. We demonstrate that for practical scenarios where the amount of radiated power and/or the number of reflecting elements are/is limited, the performance of an IRS-supported system can be significantly enhanced by utilizing a simple Decodeand-Forward (DF) relay. Tight upper bounds for the ergodic capacity are derived for the proposed scheme under different channel environments, and shown to closely match Monte-Carlo simulations.
\end{abstract}

Index Terms-Intelligent Reflecting Surface, Decode-andForward, Power Optimization, Ergodic Capacity

\section{INTRODUCTION}

W ITH the ever-increasing demand of data traffic over wireless networks, Intelligent Reflecting Surface (IRS) technology was recently proposed as a low-cost solution to enhance the performance and capacity of communications systems by smartly reconfiguring the propagation environment of wireless channels [1], [2]. A typical IRS array contains a large number of passive reflecting elements that are integrated on a planar surface and controlled by an IRS controller. Unlike other technologies such as well-known massive multiple-input multiple-output systems, IRS does not require radio-frequency chains. Instead, it simply reflects the incident signals on its planar surface with certain amplitudes and/or phase-shifts [3], such that the reflected signals are combined constructively at the receiver side [4]. Thus, it can offer a cost-effective, reliable, and energy-efficient communication [5].

A considerable amount of work has been published recently in this area. For example, the authors in [6] studied the role of IRS to enhance the physical layer security of wireless systems; while the authors in [7] demonstrated the superiority of an IRS supported system over that which comprises only an Amplify-and-Forward relay, and they studied the joint active and passive beamforming problem in an IRS supported system. Moreover, the authors in [8] showed the robustness of an IRS against phase-shift errors; while the authors in [9] evaluated the performance of an IRS supported large-scale antenna system based on statistical properties of the Channel State Information (CSI). In [10], the authors tackled the joint

This work was supported by the Engineering and Physical Sciences Research Council under Grants EP/R006377/1 and EP/R006385/1.

Z. Abdullah, G. Chen and J. A. Chambers are with the School of Engineering, University of Leicester, Leicester, LE1 7RU, UK. Emails: \{zaid.abdullah; gaojie.chen; jonathon.chambers\} @ leicester.ac.uk.

S. Lambotharan is with the Wolfson School of Mechanical, Electrical and Manufacturing Engineering, Loughborough University, Loughborough LE11 3TU, U.K. Email: s.lambotharan@lboro.ac.uk. beamforming and phase-shift design problem utilizing deep reinforcement learning. Finally, the authors in [11] compared an IRS supported system to that with a Decode-and-Forward (DF) relay in terms of energy efficiency and minimizing the total transmit power.

Thus far, several authors have focused their research on showing the efficiency of IRSs and/or comparing their performances to well-known technologies, such as relays or multiantenna systems. Motivated by this fact, and unlike the works in [7] and [11] which deal with the IRS and the relays as two separate ways to enhance link quality, in this letter, we investigate the performance gain of a novel hybrid scheme that amalgamates the benefits of both the IRS and the relay. Our main contributions in this work are summarized as follows

- We derive tight upper bounds of the achievable rates for the proposed hybrid scheme under different practical channel environments, and demonstrate their close match to Monte-Carlo simulations.

- Under a fixed power constraint, we investigate the optimal transmission mode between our hybrid scheme and that which comprises only an IRS to support transmission.

- Our results provide an insight into the performance of such hybrid schemes in terms of power optimization, mode selection, relay placement, as well as relay/IRS pairing for future networks. Finally, the performance of the proposed scheme is also evaluated under hardware impairments and imperfect CSI.

Notations: Matrices and vectors are represented by bold-face upper-case and lower-case letters, respectively. $|x|$ and $\mathbb{E}[x]$ are the absolute and expected values of $x$, respectively. $\mathbf{a}^{T}$ is the transpose of $\mathbf{a}$; while $[\mathbf{a}]_{i}$ is the $i$ th element of $\mathbf{a}$. $\operatorname{diag}(\mathbf{a})$ is a diagonal matrix whose diagonals are the elements of $\mathbf{a}$; while $\arg (x)$ is the phase of a complex number $x$.

\section{SySTEM MODEL}

We consider a network that operates in a time division duplex scenario and comprises a source (S), DF relay (R), IRS array (I), and a destination (D) as shown in Fig. 1. Unlike the IRS which has $N$ reflecting elements, $\mathrm{S}, \mathrm{R}$ and $\mathrm{D}$ have a single omnidirectional antenna each. We assume that $\mathrm{S}$ and $\mathrm{D}$ are far apart from each other and there is no direct link between the two [12]. We start with defining $\mathbf{h}_{\mathrm{SI}} \in \mathbb{C}^{N}, h_{\mathrm{SR}} \in \mathbb{C}, \mathbf{h}_{\mathrm{IR}} \in$ $\mathbb{C}^{N}, \mathbf{h}_{\mathrm{RI}} \in \mathbb{C}^{N}, h_{\mathrm{RD}} \in \mathbb{C}$, and $\mathbf{h}_{\mathrm{ID}} \in \mathbb{C}^{N}$ as the channels between $\mathrm{S} \rightarrow \mathrm{I}, \mathrm{S} \rightarrow \mathrm{R}, \mathrm{I} \rightarrow \mathrm{R}, \mathrm{R} \rightarrow \mathrm{I}^{1}, \mathrm{R} \rightarrow \mathrm{D}$, and $\mathrm{I} \rightarrow$

\footnotetext{
${ }^{1}$ Since the transmission from $\mathrm{R}$ to I occurs in a different time slot than that from I to $\mathrm{R}$, the two corresponding channel vectors are assumed to be independent from each other.
} 
$\mathrm{D}$, respectively, and each link undergoes both small and large scale fading ${ }^{2}$. During the first hop, $\mathrm{S}$ transmits its signal to both $\mathrm{R}$ and I, where the latter reflects the incident signal towards $\mathrm{R}$. We assume that the power of signals that are reflected by the IRS more than once is very small and can be ignored [7]. Therefore, the received signal at $\mathrm{R}$ can be given as

$$
y_{R}(n)=\sqrt{P_{1}}\left(h_{\mathrm{SR}}+\mathbf{h}_{\mathrm{SI}}^{T} \Theta \mathbf{h}_{\mathrm{IR}}\right) x(n)+w_{R}(n),
$$

where $n$ is the time index, $P_{1}$ is the transmit power at $\mathrm{S}$, $x(n)$ is the information symbol with $\mathbb{E}\left[|x|^{2}\right]=1, w_{R}(n)$ is the additive white Gaussian noise (AWGN) at $\mathrm{R}$ with zero mean and variance of $\sigma_{w}^{2}$, and $\Theta$ is a matrix that controls the reflection coefficients and phase-shifts of the IRS elements, and it can be expressed as $\Theta=\operatorname{diag}\left(\eta_{1} e^{j \theta_{1}}, \ldots, \eta_{N} e^{j \theta_{N}}\right)$, where $\eta_{i} \in[0,1]$ and $\theta_{i} \in[0,2 \pi]$ are the reflection coefficient and phase-shift for the $i$ th reflecting element at the IRS, respectively. Therefore, the received Signal-to-Noise Ratio (SNR) at the relay can be given as

$$
\gamma_{R}=\frac{P_{1}\left|h_{\mathrm{SR}}+\sum_{i=1}^{N} \eta_{i} e^{j \theta_{i}}\left[\mathbf{h}_{\mathrm{SI}}\right]_{i}\left[\mathbf{h}_{\mathrm{IR}}\right]_{i}\right|^{2}}{\sigma_{w}^{2}} .
$$

At time $(n+1), \mathrm{R}$ transmits the decoded signal to I and $\mathrm{D}$, where I reflects the incident signal towards $\mathrm{D}$ to be added constructively with the direct link from R. Therefore, after successful decoding of $x(n)$ at $\mathrm{R}$, the received signal at $\mathrm{D}$ can be given as

$$
y_{D}(n+1)=\sqrt{P_{2}}\left(h_{\mathrm{RD}}+\mathbf{h}_{\mathrm{RI}}^{T} \Phi \mathbf{h}_{\mathrm{ID}}\right) x(n)+w_{D}(n+1),
$$

where $P_{2}$ is the transmit power at $\mathrm{R}, w_{D}(n+1)$ is the AWGN at D with zero mean and variance of $\sigma_{w}^{2}$, and $\Phi=$ $\operatorname{diag}\left(\zeta_{1} e^{j \phi_{1}}, \ldots, \zeta_{N} e^{j \phi_{N}}\right)$ is the diagonal matrix that controls the IRS operation during the second hop, where $\zeta_{i}$ and $\phi_{i}$ are the reflection coefficient and phase-shift for the $i$ th reflecting element, respectively. Accordingly, the SNR received at the destination can be given as

$$
\gamma_{D}=\frac{P_{2}\left|h_{\mathrm{RD}}+\sum_{i=1}^{N} \zeta_{i} e^{j \phi_{i}}\left[\mathbf{h}_{\mathrm{RI}}\right]_{i}\left[\mathbf{h}_{\mathrm{ID}}\right]_{i}\right|^{2}}{\sigma_{w}^{2}} .
$$

Therefore, the achievable rate for the proposed scheme can be given as

$$
\mathcal{R}=\frac{1}{2} \log _{2}\left(1+\min \left\{\gamma_{R}, \gamma_{D}\right\}\right) .
$$

To have a fair comparison between an IRS-only-supported system and the proposed hybrid scheme, we restraint the transmission power at $\mathrm{S}$ and $\mathrm{R}$ to follow the constraint $P_{1}+P_{2}=P$, where $P$ is the total available power for the system.

In contrast, for a system that only comprises an IRS to support data transmission and has a reflection coefficient matrix $\boldsymbol{\Gamma}=\operatorname{diag}\left\{\mu_{1} e^{j \lambda_{1}}, \ldots, \mu_{N} e^{j \lambda_{N}}\right\}$, where $\mu_{i} \in[0,1]$ and $\lambda_{i} \in$ $[0,2 \pi], \forall i$, the achievable rate can be expressed as follows

$$
\mathcal{R}_{\mathrm{IRS}}=\log _{2}\left(1+\frac{P}{\sigma_{w}^{2}}\left|\sum_{i=1}^{N} \mu_{i} e^{j \lambda_{i}}\left[\mathbf{h}_{\mathrm{SI}}\right]_{i}\left[\mathbf{h}_{\mathrm{ID}}\right]_{i}\right|^{2}\right) .
$$

From (2) and (4), it is apparent that for any number of reflecting elements $N>0$, the achievable rate of the hybrid

\footnotetext{
${ }^{2}$ In this work we consider both Rayleigh and Rician fading channels from and to the IRS as will be explained in detail and analyzed in Section III.
}

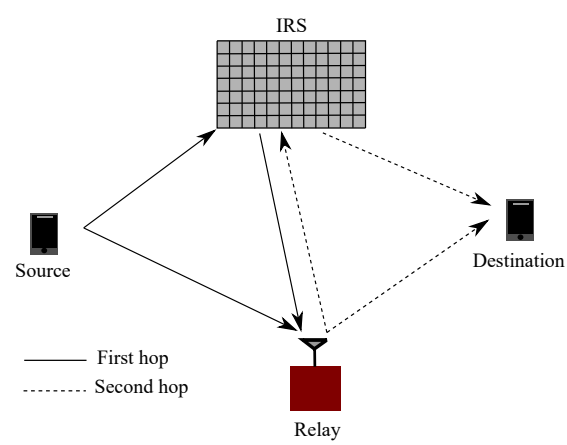

Fig. 1: The proposed system model with a source, relay, IRS, and destination.

relay-IRS scheme is always better than that of the relay only scheme, as long as the phase-shifts are properly optimized. However, it is not clear whether or not utilizing a relay can enhance the rate of an IRS-only-supported system under a fixed power constraint. This question will be addressed in the next section, where we also analyze the performance of such a hybrid scheme under different channel environments.

\section{PERformance AnAlysis AND OPtimization of the PROPOSED HYBRID SCHEME}

In this section, we first derive tight upper bounds for the achievable rates under different channel environments, and then address the optimal transmission mode selection, before carrying out the power optimization.

To simplify the analysis, Ideal Passive Beamforming (IPB) with Perfect Channel Estimation (PCE) is assumed at the IRS [7], and all elements have the same reflection amplitude ${ }^{3}$. Therefore, in (2) we have the phase $\theta_{i}=\arg \left(h_{\mathrm{SR}}\right)-$ $\arg \left(\left[\mathbf{h}_{\mathrm{SI}}\right]_{i}\left[\mathbf{h}_{\mathrm{IR}}\right]_{i}\right)$, and $\eta_{i}=\eta, \forall i$; while in (4) we have $\phi_{i}=$ $\arg \left(h_{\mathrm{RD}}\right)-\arg \left(\left[\mathbf{h}_{\mathrm{RI}}\right]_{i}\left[\mathbf{h}_{\mathrm{ID}}\right]_{i}\right)$, and $\zeta_{i}=\zeta, \forall i$. Accordingly, (2) and (4) can be simplified to

$$
\begin{aligned}
\gamma_{R} & =\frac{P_{1}\left(\left|h_{\mathrm{SR}}\right|+\eta \sum_{i=1}^{N}\left|\left[\mathbf{h}_{\mathrm{SI}}\right]_{i}\left[\mathbf{h}_{\mathrm{IR}}\right]_{i}\right|\right)^{2}}{\sigma_{w}^{2}}, \\
\gamma_{D} & =\frac{P_{2}\left(\left|h_{\mathrm{RD}}\right|+\zeta \sum_{i=1}^{N}\left|\left[\mathbf{h}_{\mathrm{RI}}\right]_{i}\left[\mathbf{h}_{\mathrm{ID}}\right]_{i}\right|\right)^{2}}{\sigma_{w}^{2}},
\end{aligned}
$$

respectively. However, the effect of discrete phase shifts and imperfect CSI will be evaluated in Section IV.

The ergodic capacity of the proposed scheme is derived by analyzing the statistics of the CSI, and then upper bounding with Jensen's inequality formula which states that

$$
\mathbb{E}\left[\log _{2}(1+v)\right] \leq \log _{2}(1+\mathbb{E}[v]) .
$$

Moreover, we consider two different types of fading for the channels between $\mathrm{S} \rightarrow \mathrm{I}, \mathrm{I} \leftrightarrow \mathrm{R}$ and $\mathrm{I} \rightarrow \mathrm{D}^{4}$. Namely, Rician

\footnotetext{
${ }^{3}$ The maximum rate is obtained when the amplitude of each reflection coefficient is set to unity, which represents an ideal scenario. However, in practical scenarios, the reflection coefficients are usually less than 1 , and can also depend on the values of the phase shifts, as demonstrated in [13].

${ }^{4}$ Links between $\mathrm{S} \rightarrow \mathrm{R}$ and $\mathrm{R} \rightarrow \mathrm{D}$ are assumed to experience Rayleigh fading at all times as they can be mobile users in a dense urban network for example, and therefore it is not guaranteed to have a LoS signal between them. However, as IRS can be installed intentionally with a clear LoS, we consider both Rayleigh and Rician fading for channels to and from the IRS.
} 


$$
\begin{aligned}
& \mathbb{E}\left[\tilde{\gamma}_{R}\right]=\mathbb{E}\left[\gamma_{1}\left(\sqrt{\tilde{\beta}_{\mathrm{SR}}}+N \sqrt{\tilde{\beta}_{\mathrm{SIR}}}\right)^{2}\right]=\gamma_{1}\left(\mathbb{E}\left[\tilde{\beta}_{\mathrm{SR}}\right]+2 N \mathbb{E}\left[\sqrt{\tilde{\beta}_{\mathrm{SR}}} \sqrt{\tilde{\beta}_{\mathrm{SIR}}}\right]+N^{2} \mathbb{E}\left[\tilde{\beta}_{\mathrm{SIR}}\right]\right) \\
& \leq \gamma_{1}\left(d_{\mathrm{SR}}^{-\alpha}+\frac{1}{2} d_{\mathrm{SR}}^{-\frac{\alpha}{2}} d_{\mathrm{SI}}^{-\frac{\alpha}{2}} d_{\mathrm{IR}}^{-\frac{\alpha}{2}} \eta \sqrt{N} \sqrt{16+(N-1) \pi^{2}}+\frac{1}{16} d_{\mathrm{SI}}^{-a} d_{\mathrm{IR}}^{-a} \eta^{2} N\left(16+(N-1) \pi^{2}\right)\right), \\
& \mathbb{E}\left[\tilde{\gamma}_{D}\right] \leq \gamma_{2}\left(d_{\mathrm{RD}}^{-\alpha}+\frac{1}{2} d_{\mathrm{RD}}^{-\frac{\alpha}{2}} d_{\mathrm{IR}}^{-\frac{\alpha}{2}} d_{\mathrm{ID}}^{-\frac{\alpha}{2}} \zeta \sqrt{N} \sqrt{16+(N-1) \pi^{2}}+\frac{1}{16} d_{\mathrm{IR}}^{-a} d_{\mathrm{ID}}^{-a} \zeta^{2} N\left(16+(N-1) \pi^{2}\right)\right),
\end{aligned}
$$

fading channels with pure Line-of-Sight (LoS) components ${ }^{5}$, and Non-Line-of-Sight (NLoS) Rayleigh fading channels. We will refer to the two different types of channels throughout our analysis as follows

$$
\mathbf{h}_{j}= \begin{cases}\sqrt{\frac{K_{j}}{K_{j}+1}} \overline{\mathbf{h}}_{j}, & \text { for } \operatorname{LoS} \text { (Rician) } \\ \tilde{\mathbf{h}}_{j}, & \text { for NLoS (Rayleigh) }\end{cases}
$$

where $K_{j}$ is the Rician $K$-factor, $j \in\{\mathrm{SI}, \mathrm{IR}, \mathrm{RI}, \mathrm{ID}\}$.

\section{A. A tight upper bound for the achievable rate with Rayleigh fading channels}

In this case, only NLoS channels exist and the links can be expressed as $\tilde{\mathbf{h}}_{j}=\tilde{\mathbf{g}}_{j} d_{j}^{-\alpha / 2}, j \in\{\mathrm{SI}, \mathrm{IR}, \mathrm{RI}, \mathrm{ID}\}$, and $\tilde{h}_{k}=\tilde{g}_{k} d_{k}^{-\alpha / 2}, k \in\{\mathrm{SR}, \mathrm{RD}\}$, where $\tilde{g} \in \mathbb{C}$ and $\tilde{\mathbf{g}} \in \mathbb{C}^{N}$ are the complex-Gaussian small-scale fading channels with zero mean and unit variance, $d$ is the distance between two nodes, and $\alpha$ is the path-loss exponent.

We define $\sqrt{\tilde{\beta}_{\mathrm{SR}}}=\left|\tilde{h}_{\mathrm{SR}}\right|, \sqrt{\tilde{\beta}_{\mathrm{SIR}}}=\frac{\eta}{N} \sum_{i=1}^{N}\left|\left[\tilde{\mathbf{h}}_{\mathrm{SI}}\right]_{i}\left[\tilde{\mathbf{h}}_{\mathrm{IR}}\right]_{i}\right|$, $\sqrt{\tilde{\beta}_{\mathrm{RID}}}=\frac{\zeta}{N} \sum_{i=1}^{N}\left|\left[\tilde{\mathbf{h}}_{\mathrm{RI}}\right]_{i}\left[\tilde{\mathbf{h}}_{\mathrm{ID}}\right]_{i}\right|, \sqrt{\tilde{\beta}_{\mathrm{RD}}}=\left|\tilde{h}_{\mathrm{RD}}\right|$, and $\gamma_{l}=$ $P_{l} / \sigma_{w}^{2}, l \in\{1,2\}$. Both $\tilde{\beta}_{\mathrm{SR}}$ and $\tilde{\beta}_{\mathrm{RD}}$ are exponentially distributed with $\mathbb{E}\left[\tilde{\beta}_{\mathrm{SR}}\right]=d_{\mathrm{SR}}^{-\alpha}$ and $\mathbb{E}\left[\tilde{\beta}_{\mathrm{RD}}\right]=d_{\mathrm{RD}}^{-\alpha}$. In contrast, and for a large number of reflecting elements $N, \tilde{\beta}_{\text {SIR }}$ and $\tilde{\beta}_{\text {RID }}$ follow a non-central chi-square distribution with $\mathbb{E}\left[\tilde{\beta}_{\mathrm{SIR}}\right]=\frac{\eta^{2}\left[\pi^{2}+(1 / N)\left(16-\pi^{2}\right)\right]}{16 d_{\mathrm{SI}}^{\alpha} d_{\mathrm{IR}}^{\alpha}}$ and $\mathbb{E}\left[\tilde{\beta}_{\mathrm{RID}}\right]=$ $\frac{\zeta^{2}\left[\pi^{2}+(1 / N)\left(16-\pi^{2}\right)\right]}{16 d_{\mathbb{R}}^{\alpha} d_{\mathrm{N}}^{\alpha}}[15]$. Therefore, the expected value of $\tilde{\gamma}_{R}$ can be derived as shown in (10). It should be noted that the second term in (a) holds by applying Holder inequality such that $\mathbb{E}[|x y|] \leq\left(\mathbb{E}\left[|x|^{v}\right]\right)^{1 / v}\left(\mathbb{E}\left[|y|^{q}\right]\right)^{1 / q}$, where $x$ and $y$ are real or complex-valued random variables, $v, q \geq 1$, and $1 / v+1 / q=1$ (in our case $v=q=2$ ). Moreover, and after following the same steps in (10), the expected value of $\tilde{\gamma}_{D}$ is given in (11). Thus, the upper bound ergodic capacity for the proposed scheme with Rayleigh fading channels can be given as

$$
\tilde{\mathcal{R}}_{\text {upper }}=\frac{1}{2} \log _{2}\left(1+\min \left\{\mathbb{E}\left[\tilde{\gamma}_{R}\right], \mathbb{E}\left[\tilde{\gamma}_{D}\right]\right\}\right) .
$$

\section{B. A tight upper bound for the achievable rate with mixed Rayleigh and Rician fading channels}

In this case, channels from and to the IRS can be given as $\overline{\mathbf{h}}_{i}=\overline{\mathbf{g}}_{i} d_{i}^{-\alpha / 2}, i \in\{\mathrm{SI}, \mathrm{IR}, \mathrm{RI}, \mathrm{ID}\}$, where $\overline{\mathbf{g}}_{i}=$ $\left[e^{j \psi_{i, 1}}, \ldots ., e^{j \psi_{i, N}}\right]^{T}$ is a fixed-component vector with elements of unit power, and $\psi_{i, n} \in[0,2 \pi]$ is the phase for the

\footnotetext{
${ }^{5}$ In a Rician fading environment, the LoS component becomes dominant and therefore the NLoS channel can be ignored [14].
}

$n$th channel, which is determined by wave-length, distance between reflecting elements, and angle of arrival/departure ${ }^{6}$.

We let $\sqrt{\bar{\beta}_{\mathrm{SIR}}}=\frac{\eta}{N} \delta_{\mathrm{SIR}} \sum_{i=1}^{N}\left|\left[\overline{\mathbf{h}}_{\mathrm{SI}}\right]_{i}\left[\overline{\mathbf{h}}_{\mathrm{IR}}\right]_{i}\right|$ and $\sqrt{\bar{\beta}_{\mathrm{RID}}}=$ $\frac{\zeta}{N} \delta_{\mathrm{RID}} \sum_{i=1}^{N}\left|\left[\overline{\mathbf{h}}_{\mathrm{RI}}\right]_{i}\left[\overline{\mathbf{h}}_{\mathrm{ID}}\right]_{i}\right|$, where $\delta_{\mathrm{SIR}}=\sqrt{\frac{K_{\mathrm{SI}} K_{\mathrm{IR}}}{\left(K_{\mathrm{SI}}+1\right)\left(K_{\mathrm{IR}}+1\right)}}$, and $\delta_{\mathrm{RID}}=\sqrt{\frac{K_{\mathrm{II}} K_{\mathrm{ID}}}{\left(K_{\mathrm{IR}}+1\right)\left(K_{\mathrm{ID}}+1\right)}}$; while $\sqrt{\tilde{\beta}_{\mathrm{SR}}}$ and $\sqrt{\tilde{\beta}_{\mathrm{RD}}}$ remain the same since they experience Rayleigh fading as mentioned previously, with $\mathbb{E}\left[\sqrt{\tilde{\beta}_{\mathrm{SR}}}\right]=\sqrt{\frac{\pi}{4 d_{\mathrm{SR}}^{\alpha}}}$ and $\mathbb{E}\left[\sqrt{\tilde{\beta}_{\mathrm{RD}}}\right]=\sqrt{\frac{\pi}{4 d_{\mathrm{RD}}^{\alpha}}}$. Moreover, $\bar{\beta}_{\mathrm{SIR}}$ and $\bar{\beta}_{\mathrm{RID}}$ have constant mean values of $\mathbb{E}\left[\bar{\beta}_{\mathrm{SIR}}\right]=\frac{\eta^{2} \delta_{\mathrm{SR}}^{2}}{d_{\mathrm{SI}}^{\alpha} d_{\mathrm{TR}}^{\alpha}}$ and $\mathbb{E}\left[\bar{\beta}_{\mathrm{RID}}\right]=\frac{\zeta^{2} \delta_{\mathrm{RID}}^{2}}{d_{\mathrm{IR}}^{\alpha} d_{\mathrm{ID}}^{\alpha}}$. Therefore, the expected value of the received SNR at the relay with mixed fading channels $\hat{\gamma}_{R}$, can be derived as follows

$$
\begin{aligned}
\mathbb{E}\left[\hat{\gamma}_{R}\right] & =\mathbb{E}\left[\gamma_{1}\left(\sqrt{\tilde{\beta}_{\mathrm{SR}}}+N \sqrt{\bar{\beta}_{\mathrm{SIR}}}\right)^{2}\right] \\
& =\gamma_{1}\left(\mathbb{E}\left[\tilde{\beta}_{\mathrm{SR}}\right]+\eta \frac{2 N \delta_{\mathrm{SIR}}}{d_{\mathrm{SI}}^{\frac{\alpha}{2}} d_{\mathrm{IR}}^{\frac{\alpha}{2}}} \mathbb{E}\left[\sqrt{\tilde{\beta}_{\mathrm{SR}}}\right]+\eta^{2} \frac{N^{2} \delta_{\mathrm{SIR}}^{2}}{d_{\mathrm{SI}}^{\alpha} d_{\mathrm{IR}}^{\alpha}}\right) \\
& =\gamma_{1}\left(d_{\mathrm{SR}}^{-\alpha}+\eta \frac{\sqrt{\pi} N \delta_{\mathrm{SIR}}}{d_{\mathrm{SR}}^{\frac{\alpha}{2}} d_{\mathrm{SI}}^{\frac{\alpha}{2}} d_{\mathrm{IR}}^{\frac{\alpha}{2}}}+\eta^{2} \frac{N^{2} \delta_{\mathrm{SIR}}^{2}}{d_{\mathrm{SI}}^{\alpha} d_{\mathrm{IR}}^{\alpha}}\right) .
\end{aligned}
$$

Similarly, the expected value of $\hat{\gamma}_{D}$ can be given as follows

$$
\mathbb{E}\left[\hat{\gamma}_{D}\right]=\gamma_{2}\left(d_{\mathrm{RD}}^{-\alpha}+\zeta \frac{\sqrt{\pi} N \delta_{\mathrm{RID}}}{d_{\mathrm{RD}}^{\frac{\alpha}{2}} d_{\mathrm{IR}}^{\frac{\alpha}{2}} d_{\mathrm{ID}}^{\frac{\alpha}{2}}}+\zeta^{2} \frac{N^{2} \delta_{\mathrm{RID}}^{2}}{d_{\mathrm{IR}}^{\alpha} d_{\mathrm{ID}}^{\alpha}}\right) .
$$

Accordingly, the upper bound for the ergodic capacity with mixed fading channels can be expressed as

$$
\hat{\mathcal{R}}_{\text {upper }}=\frac{1}{2} \log _{2}\left(1+\min \left\{\mathbb{E}\left[\hat{\gamma}_{R}\right], \mathbb{E}\left[\hat{\gamma}_{D}\right]\right\}\right) .
$$

\section{Optimal power allocation and transmission mode selection}

In this subsection, we analyze the optimal mode selection based on Equal Power Allocation (EPA), and then carry out an Optimal Power Allocation (OPA) at the source and relay to maximize the achievable rate.

1) Optimal mode selection: we first define the channel gains for a system that only comprises an IRS to support data transmission as follows ${ }^{7}:$ let $\sqrt{\tilde{\beta}_{\mathrm{SID}}}=\frac{\mu}{N} \sum_{i=1}^{N}\left|\left[\tilde{\mathbf{h}}_{\mathrm{SI}}\right]_{i}\left[\tilde{\mathbf{h}}_{\mathrm{ID}}\right]_{i}\right|$, and $\sqrt{\bar{\beta}_{\mathrm{SID}}}=\frac{\mu}{N} \delta_{\mathrm{SID}} \sum_{i=1}^{N}\left|\left[\overline{\mathbf{h}}_{\mathrm{SI}}\right]_{i}\left[\overline{\mathbf{h}}_{\mathrm{ID}}\right]_{i}\right|$ be the channel gains for an IRS-only-supported system over Rayleigh and Rician fading channels, respectively, where $\delta_{\mathrm{SID}}=\sqrt{\frac{K_{\mathrm{SI}} K_{\mathrm{ID}}}{\left(K_{\mathrm{SI}}+1\right)\left(K_{\mathrm{ID}}+1\right)}}$.

\footnotetext{
${ }^{6}$ In our work, the phase of each link for the Rician channels was randomly generated between 0 and $2 \pi$ as in [9].

${ }^{7} \mathrm{We}$ assume IPB for the system in (6), such that $\lambda_{i}=$ $-\arg \left(\left[\mathbf{h}_{\mathrm{SI}}\right]_{i}\left[\mathbf{h}_{\mathrm{ID}}\right]_{i}\right), \forall i$, and all elements have the same reflection amplitude, i.e. $\mu_{i}=\mu, \forall i$.
} 


$$
P_{\min } \geq \min \left\{\frac{\left[\tilde{\beta}_{\mathrm{SR}}+\left(\frac{2}{N} \sqrt{\tilde{\beta}_{\mathrm{SR}}} \sqrt{\dot{\beta}_{\mathrm{SIR}}}+\dot{\beta}_{\mathrm{SIR}}-4 \dot{\beta}_{\mathrm{SID}}\right) N^{2}\right] \sigma_{w}^{2}}{2 N^{4} \dot{\beta}_{\mathrm{SID}}^{2}}, \frac{\left[\tilde{\beta}_{\mathrm{RD}}+\left(\frac{2}{N} \sqrt{\tilde{\beta}_{\mathrm{RD}}} \sqrt{\dot{\beta}_{\mathrm{RID}}}+\dot{\beta}_{\mathrm{RID}}-4 \dot{\beta}_{\mathrm{SID}}\right) N^{2}\right] \sigma_{w}^{2}}{2 N^{4} \dot{\beta}_{\mathrm{SID}}^{2}}\right\} .
$$

$$
\mathcal{R}_{\max }=\frac{1}{2} \log _{2}\left\{1+\frac{P}{\sigma_{w}^{2}}\left(\frac{\left(\tilde{\beta}_{\mathrm{SR}}+2 N \sqrt{\tilde{\beta}_{\mathrm{SR}}} \sqrt{\dot{\beta}_{\mathrm{SIR}}}+N^{2} \dot{\beta}_{\mathrm{SIR}}\right)\left(\tilde{\beta}_{\mathrm{RD}}+2 N \sqrt{\tilde{\beta}_{\mathrm{RD}}} \sqrt{\dot{\beta}_{\mathrm{RID}}}+N^{2} \dot{\beta}_{\mathrm{RID}}\right)}{\tilde{\beta}_{\mathrm{SR}}+\tilde{\beta}_{\mathrm{RD}}+2 N\left(\sqrt{\tilde{\beta}_{\mathrm{SR}}} \sqrt{\dot{\beta}_{\mathrm{SIR}}}+\sqrt{\tilde{\beta}_{\mathrm{RD}}} \sqrt{\dot{\beta}_{\mathrm{RID}}}\right)+N^{2}\left(\dot{\beta}_{\mathrm{SIR}}+\dot{\beta}_{\mathrm{RID}}\right)}\right)\right\} .
$$

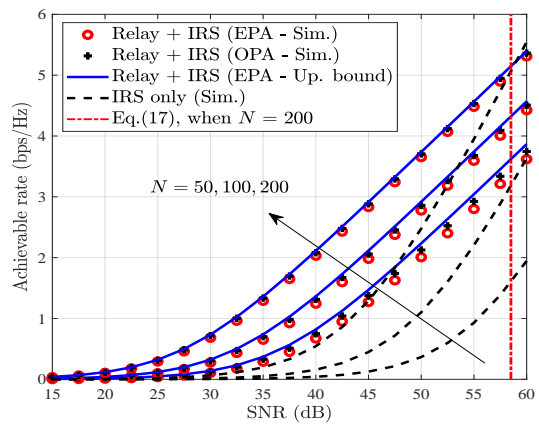

Fig. 2: Achievable rate vs SNR under Rayleigh fading channels and for different values of $N$.

Then, by utilizing (5) and (6), and after mathematical manipulations, the minimum required transmit power for an IRSonly-supported system to outperform the hybrid scheme can be given as shown in (17), where $\dot{\beta}_{j} \in\left\{\tilde{\beta}_{j}, \bar{\beta}_{j}\right\}$, depending whether a specific link $j$ between two nodes is LoS or NLoS, respectively. Note that the value of $P_{\min }$ can be negative, in which case a system with only an IRS will always outperform the hybrid scheme. Our first intuition is that for realistic values of $N$, the transmit $\operatorname{SNR}\left(P_{\min } / \sigma_{w}^{2}\right)$ needs to be extremely high to outperform the hybrid scheme, as the value of $\dot{\beta}_{\text {SID }}^{2}$ is usually extremely small.

2) Optimal power allocation: for systems with more than one hop, power optimization can lead to a better performance. Moreover, since we consider a DF relay, the maximum rate can be achieved when the received SNR at the relay is equal to that at the destination, i.e. $\tilde{\gamma}_{R}=\tilde{\gamma}_{D}$ and $\hat{\gamma}_{R}=\hat{\gamma}_{D}$, for Rayleigh and mixed fading channels, respectively. Considering a total power constraint of $P_{1}+P_{2}=P$, and after solving some mathematical equations, the maximum rate with OPA for the proposed scheme is given in (18).

\section{RESULTS AND DISCUSSION}

Unless stated otherwise, the source was located at the origin, i.e. $\left(x_{\mathrm{S}}, y_{\mathrm{S}}\right)=(0,0)$, while $\left(x_{\mathrm{I}}, y_{\mathrm{I}}\right)=(50,10)$, $\left(x_{\mathrm{R}}, y_{\mathrm{R}}\right)=(50,0)$, and $\left(x_{\mathrm{D}}, y_{\mathrm{D}}\right)=(100,0)$. $\alpha$ was set to 2 for LoS links, and 2.5 for NLoS channels, $\sigma_{w}^{2}$ was set to 1 , while the Rician- $K$ factor for all links from and to the IRS was $10 \mathrm{~dB}$. All reflection coefficients $\left(\eta_{i}, \zeta_{i}, \mu_{i}\right)$ were set to 0.8 , $\forall i$. Finally, the SNR is defined as $P / \sigma_{w}^{2}$, and the simulations were averaged over $10^{4}$ different channel realizations.

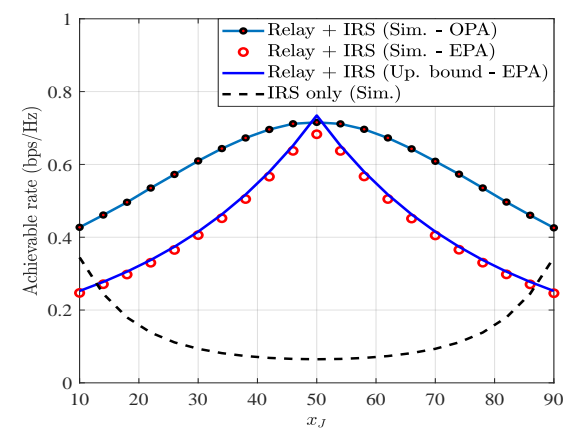

Fig. 3: Achievable rate under Rayleigh fading when $x_{\mathrm{I}}=x_{\mathrm{R}}=$ $x_{\mathrm{J}}, N=200$, and SNR is $30 \mathrm{~dB}$.

\section{A. Achievable rates in an ideal environment}

Here we demonstrate the efficiency of the proposed scheme in an ideal scenario, where we have IPB and PCE.

Fig. 2 demonstrates the achievable rate over a wide range of SNR values. The hybrid scheme shows a significant gain, under the same total power constraint, compared to a system that comprises only an IRS array. For example, when the number of reflecting elements is 200 , to achieve a rate of 2 bps/Hz, the combined $\mathrm{R}$ and IRS scheme requires $40 \mathrm{~dB}$ of total transmit SNR; while the same rate comes at $48 \mathrm{~dB}$ of transmit power for a system with only an IRS. Moreover, as (17) indicates, an IRS-only-supported system outperforms the hybrid scheme only when the number of reflecting elements is large and over the high SNR region.

Meanwhile, in Fig. 3 we consider different locations of both relay and IRS in terms of $x_{\mathrm{R}}$ and $x_{\mathrm{I}}$, while keeping the distance between the two fixed (i.e. $d_{\mathrm{IR}}$ is fixed). The IRS on its own performs best when it is close to either the source or destination. In contrast, when combined with a relay, the optimal locations for the two nodes are in the middle between the source and destination. Moreover, OPA is of significant importance when the relay is close to either the source or destination, while EPA becomes near optimal when the relay and IRS are located in the middle between the two ends.

Fig. 4 demonstrates the performance of the hybrid scheme and that which only considers an IRS under mixed Rician and Rayleigh fading channels, for a wide range of $N$ values. Once again, the proposed scheme shows a great advantage over the IRS-only scheme especially at low to moderate SNRs. For example, to achieve a rate of $2 \mathrm{bps} / \mathrm{Hz}$, and when the transmit 


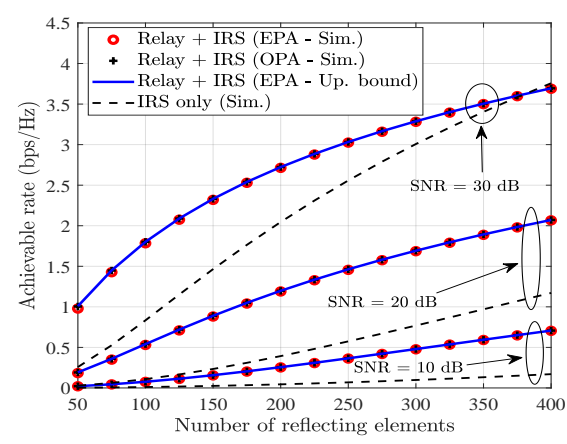

Fig. 4: The achievable rate for a wide range of values of $N$ under mixed Rician and Rayleigh fading environment.

SNR is $30 \mathrm{~dB}$, the combined R and IRS scheme requires an IRS with 118 reflecting elements, compared to 196 elements that the IRS demands to achieve the same rate on its own.

\section{B. Discrete phase-shifts and imperfect CSI}

In practical implementations, ideal (continuous) phase shifts may be difficult or add considerable complexity. Instead, discrete phase-shifts are used where each reflecting element can only take a value from a finite set of discrete phaseshift values. Let $\mathcal{F} \in\left\{0, \frac{2 \pi}{K}, \ldots, \frac{(K-1) 2 \pi}{K}\right\}$ be a set with $K$ uniformly quantized phase-shift levels, and the discrete phase-shift of each reflecting element is the quantization of its continuous phase-shift value to the nearest point in $\mathcal{F}$.

Moreover, the inevitable channel estimation errors will lead to phase-shift errors at the IRS. Assuming Rayleigh fading channels, we denote the estimated channels at the IRS by $\hat{\mathbf{h}}_{k}=\sqrt{1-\varepsilon} \tilde{\mathbf{h}}_{k}+\sqrt{\varepsilon} \check{\mathbf{h}}_{k}$, for $k \in\{$ SI, IR, RI, ID $\}$, and $\hat{h}_{j}=\sqrt{1-\varepsilon} \tilde{h}_{j}+\sqrt{\varepsilon} \tilde{h}_{j}$, for $j \in\{\mathrm{SR}, \mathrm{RD}\}$, where $\varepsilon \in[0,1]$ accounts for the estimation accuracy, which depends on the utilized channel estimation method, $\breve{\mathbf{h}}_{k}$ and $\breve{h}_{j}$ are the estimation errors, which are uncorrelated with $\tilde{\mathbf{h}}_{k}$ and $\tilde{h}_{j}$, but have the same statistical properties as of $\tilde{\mathbf{h}}_{k}$ and $\tilde{h}_{j}$ in terms of mean and variance values.

As demonstrated in Fig.5, both the discrete phase-shifts and imperfect CSI result in a degraded performance. However, both the hybrid scheme and the IRS-only scheme show robustness against imperfect CSI and non-ideal passive beamforming, and the hybrid scheme with EPA achieves the highest rates for the whole range of considered values of SNR.

\section{CONCLUSIONS}

The benefits of combining a classical DF relay with an IRS array was evaluated in this work and compared to the case in which a system utilized only an IRS surface to support data transmission. Tight upper bounds for the achievable rates were derived and shown to closely match the MonteCarlo simulations under different environments. Our work demonstrated that for low and moderate SNRs, using a singleantenna DF relay can save a massive number of reflecting elements to achieve the same performance, and at no additional power cost; while a system with only an IRS can be optimal

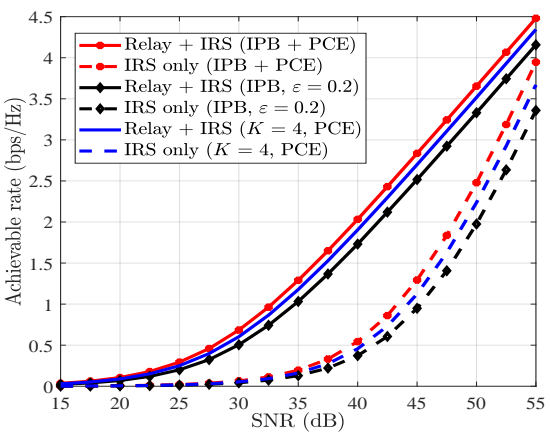

Fig. 5: The effects of discrete phase-shifts and imperfect CSI under Rayleigh fading channels when $N=200$.

only when the SNR is very high and the number of reflecting elements is extremely large.

\section{REFERENCES}

[1] Q. Wu and R. Zhang, "Towards smart and reconfigurable environment: Intelligent reflecting surface aided wireless network," IEEE Commun. Mag., vol. 58, pp. 106-112, Jan. 2020.

[2] C. Huang, S. Hu, G. C. Alexandropoulos, A. Zappone, C. Yuen, R. Zhang, M. Di Renzo, and M. Debbah, "Holographic MIMO surfaces for 6G wireless networks: Opportunities, challenges, and trends," [Online]. Available: https://arxiv.org/abs/1911.12296, 2019.

[3] X. Tan, Z. Sun, J. M. Jornet, and D. Pados, "Increasing indoor spectrum sharing capacity using smart reflect-array," in IEEE Inter. Conf. Commun. (ICC), Kuala Lumpur, Malaysia, May 2016, pp. 1-6.

[4] C. Huang, A. Zappone, G. C. Alexandropoulos, M. Debbah, and C. Yuen, "Reconfigurable intelligent surfaces for energy efficiency in wireless communication," IEEE Trans. Wireless Commun., vol. 18, no. 8, pp. 4157-4170, Aug. 2019.

[5] Q. Wu and R. Zhang, "Beamforming optimization for wireless network aided by intelligent reflecting surface with discrete phase shifts," IEEE Trans. Commun., To appear. 2020.

[6] M. Cui, G. Zhang, and R. Zhang, "Secure wireless communication via intelligent reflecting surface," IEEE Wireless Commun. Lett., vol. 8, no. 5, pp. 1410-1414, Oct. 2019.

[7] Q. Wu and R. Zhang, "Intelligent reflecting surface enhanced wireless network via joint active and passive beamforming," IEEE Trans. Wireless Commun., vol. 18, no. 11, pp. 5394-5409, Nov. 2019.

[8] M.-A. Badiu and J. P. Coon, "Communication through a large reflecting surface with phase errors," IEEE Wireless Commun. Lett., vol. 9, no. 2, pp. 184-188, Feb. 2020.

[9] Y. Han, W. Tang, S. Jin, C.-K. Wen, and X. Ma, "Large intelligent surface-assisted wireless communication exploiting statistical CSI," IEEE Trans. Veh. Technol., vol. 68, no. 8, pp. 8238-8242, Aug. 2019.

[10] C. Huang, R. Mo, and C. Yuen, "Reconfigurable intelligent surface assisted multiuser MISO systems exploiting deep reinforcement learning," [Online]. Available: https://arxiv.org/abs/2002.10072.

[11] E. Björnson, Ö. Özdogan, and E. G. Larsson, "Intelligent reflecting surface vs. decode-and-forward: How large surfaces are needed to beat relaying?" IEEE Wireless Commun. Lett., vol. 9, no. 2, pp. 244-248, Feb. 2020.

[12] D. Lee and J. H. Lee, "Outage probability of decode-and-forward opportunistic relaying in a multicell environment," IEEE Trans. Veh. Technol., vol. 60, no. 4, pp. 1925-1930, May 2011.

[13] S. Abeywickrama, R. Zhang, Q. Wu, and C. Yuen, "Intelligent reflecting surface: Practical phase shift model and beamforming optimization," [Online]. Available: https://arxiv.org/abs/2002.10112.

[14] K. Kobayashi, T. Ohtsuki, and T. Kaneko, "Precoding for MIMO systems in line-of-sight (LOS) environment," in IEEE Global Commun. Conf. (GLOBECOM), Washington, DC, USA. Nov. 2007, pp. 43704374.

[15] E. Basar, M. Di Renzo, J. De Rosny, M. Debbah, M.-S. Alouini, and R. Zhang, "Wireless communications through reconfigurable intelligent surfaces," IEEE Access, vol. 7, pp. 116753-116773, Sep. 2019. 